\title{
Astragaloside IV Inhibits Oxidative Stress-Induced Mitochondrial Permeability Transition Pore Opening by Inactivating GSK-3 $\beta$ via Nitric Oxide in H9c2 Cardiac Cells
}

\author{
Yonggui He, ${ }^{1,2}$ Jinkun Xi, ${ }^{3}$ Huan Zheng, ${ }^{3}$ Yidong Zhang, ${ }^{3}$ Yuanzhe Jin, ${ }^{2}$ and Zhelong $\mathrm{Xu}^{4}$ \\ ${ }^{1}$ Department of Internal Medicine, Hebei United University, Tangshan 063000, China \\ ${ }^{2}$ Department of Physiology, Yanbian University, Yanji 133002, China \\ ${ }^{3}$ Heart Institute, Hebei United University, Tangshan 063000, China \\ ${ }^{4}$ Department of Anesthesiology, The University of North Carolina at Chapel Hill, CB No. 7010, Chapel Hill, NC 27599, USA
}

Correspondence should be addressed to Yuanzhe Jin, yz-jin@ybu.edu.cn and Zhelong Xu, zxu@aims.unc.edu

Received 19 June 2012; Accepted 13 August 2012

Academic Editor: Paola Venditti

Copyright () 2012 Yonggui He et al. This is an open access article distributed under the Creative Commons Attribution License, which permits unrestricted use, distribution, and reproduction in any medium, provided the original work is properly cited.

\begin{abstract}
Objective. This study aimed to investigate whether astragaloside IV modulates the mitochondrial permeability transition pore (mPTP) opening through glycogen synthase kinase $3 \beta$ (GSK-3 $\beta$ ) in H9c2 cells. Methods. H9c2 cells were exposed to astragaloside IV for 20 min. GSK-3 $\beta\left(\operatorname{Ser}^{9}\right)$, Akt $\left(\operatorname{Ser}^{473}\right)$, and VASP $\left(\operatorname{Ser}^{239}\right)$ activities were determined with western blot. The mPTP opening was evaluated by measuring mitochondrial membrane potential $\left(\Delta \Psi_{m}\right)$. Nitric oxide (NO) generation was measured by 4 -amino5-methylamino-2', $7^{\prime}$-difluorofluorescein (DAF-FM) diacetate. Fluorescence images were obtained with confocal microscopy. Results. Astragaloside IV significantly enhanced GSK-3 $\beta$ phosphorylation and prevented $\mathrm{H}_{2} \mathrm{O}_{2}$-induced loss of $\Delta \Psi_{m}$. These effects of astragaloside IV were reversed by the phosphatidylinositol 3-kinase (PI3K) inhibitor LY294002, the NO sensitive guanylyl cyclase selective inhibitor ODQ, and the PKG inhibitor KT5823. Astragaloside IV activated Akt and PKG. Astragaloside IV was also shown to increase NO production, an effect that was reversed by L-NAME and LY294002. Astragaloside IV applied at reperfusion reduced cell death caused by simulated ischemia/reperfusion, indicating that astragaloside IV can prevent reperfusion injury. Conclusions. These data suggest that astragaloside IV prevents the mPTP opening and reperfusion injury by inactivating GSK-3 $\beta$ through the $\mathrm{NO} / \mathrm{cGMP} / \mathrm{PKG}$ signaling pathway. NOS is responsible for NO generation and is activated by the PI3K/Akt pathway.
\end{abstract}

\section{Introduction}

As a major active ingredient of the traditional Chinese herb Radix Astragali, astragaloside IV (see Figure $1(\mathrm{~S})$ in Supplementary Material available online at doi:10.1155/2012/935738) exerts numerous biological effects [1-6]. It has been widely used for treatments of cardiovascular diseases in China from the ancient time, and its cardioprotective in canine hearts [7]. Although Ca-ATPase [8], $\mathrm{Na}(+)-\mathrm{K}(+)$-ATPase [9], antioxidant [10], nitric oxide (NO) [7] have been proposed to be involved in the action of astragaloside IV, the exact cellular and molecular mechanism by which astragaloside IV induces cardioprotection remains unclear.

The mitochondrial permeability transition pore (mPTP) plays a critical role in the pathogenesis of myocardial ischemia/reperfusion injury $[11,12]$. Inhibition of the $\mathrm{mPTP}$ opening at early reperfusion can protect the heart from reperfusion injury [13-18]. Since astragaloside IV protects the heart through a NO-dependent mechanism [7] and NO has been demonstrated to prevent the MPTP opening [19], it is possible that astragaloside IV can modulate the MPTP opening at reperfusion.

Identified originally as a regulator of glycogen metabolism, glycogen synthase kinase $3 \beta$ (GSK-3 $\beta$ ) is now a well-established component contributing to cell signaling, protein synthesis, cell proliferation, cell differentiation, cell adhesion, and apoptosis [20,21]. Studies have demonstrated that GSK- $3 \beta$ plays a role in ischemic preconditioning [22] and in morphine-induced cardioprotection at reperfusion [23]. GSK-3 $\beta$ was further proposed to play a central role in pharmacological preconditioning-induced modulation of 
the mPTP opening [24]. In addition, postconditioning [25], ethanol [26], resveratrol [27], and morphine [28] have also been reported to protect the heart by targeting the $\mathrm{mPTP}$ through inhibition of GSK-3 $\beta$. Since NO was demonstrated to be involved in the cardioprotective effect of astragaloside IV [7] and the cGMP/PKG signaling pathway can negatively regulate GSK-3 $\beta[28,29]$; since NO could protect the heart by targeting mPTP through GSK-3 $\beta[30]$, it is likely that GSK-3 $\beta$ plays a role in the action of astragaloside IV through NO signaling pathway.

This study first examined if astragaloside IV inactivates GSK-3 $\beta$ by detecting phosphorylation of GSK-3 $\beta$ at Ser ${ }^{9}$. Then experiments were conducted to determine if astragaloside IV could prevent oxidative stress-induced mPTP opening through inactivation of GSK-3 $\beta$. Lastly, the study was aimed to define the signaling mechanism by which astragaloside IV inactivates GSK-3 $\beta$, focusing on the roles of PI3 K/Akt, NO, and cGMP/PKG.

\section{Materials and Methods}

2.1. Cell Culture. The rat heart tissue-derived $\mathrm{H} 9 \mathrm{c} 2$ cardiac myoblast cell line was purchased from American Type Culture Collection (ATCC, Manassas, VA, USA). Cells were cultured in Dulbecco's modified Eagle's medium (DMEM) (Invitrogen) supplemented with $10 \%$ fetal bovine serum (FBS) (Invitrogen) and $100 \mathrm{U}$ penicillin/streptomycin at $37^{\circ} \mathrm{C}$ in a humidified $5 \% \mathrm{CO}_{2}-95 \%$ air atmosphere.

2.2. Chemicals and Antibodies. Astragaloside IV was purchased from the National Institute for the Control of Pharmaceutical and Biological Products (NICPB, Beijing, China), with a high purity $99 \%$ by HPLC analysis. Tetramethylrhodamine ethyl ester (TMRE) and 4-amino-5-methylamino- $2^{\prime}$, $7^{\prime}$-difluorofluorescein (DAF-FM) diacetate were purchased from Molecular Probes (Eugene, OR, USA). LY294002, ODQ, KT5823, SB216763, cyclosporin A, and FCCP were obtained from Sigma (St. Louis, MO, USA). All antibodies were purchased from Cell Signaling Technology (Beverly, MA, USA).

2.3. Confocal Imaging of Mitochondrial Membrane Potential. Mitochondrial membrane potential $\left(\Delta \Psi_{m}\right)$ was measured by loading H9c2 cardiac cells with TMRE using confocal microscopy as reported previously [29]. TMRE is a cell permeable, cationic, nontoxic, fluorescent dye that specifically stains live mitochondria. TMRE is accumulated specifically by the mitochondria in proportion to membrane potential [31]. A number of studies have measured $\Delta \Psi_{m}$ by imaging cardiac cells loaded with TMRE [32-34]. Briefly, cells cultured in a specific temperature-controlled culture dish (MatTek, MA, USA) were incubated with TMRE (100 nM) in standard Tyrode solution containing $(\mathrm{mM}) \mathrm{NaCl} 140$, $\mathrm{KCl} 6, \mathrm{MgCl}_{2} 1, \mathrm{CaCl}_{2} 1$, HEPES 5, and glucose $5.8(\mathrm{pH}$ 7.4) for $10 \mathrm{~min}$. Cells were washed several times with fresh Tyrode solution. The dish was then mounted on the stage of an Olympus FLUOVIEW FV 1000 laser scanning confocal microscope (Olympus Corporation, Tokyo, Japan). The red fluorescence was excited with a $543 \mathrm{~nm}$ line of He-Ne laser line and imaged through a $560 \mathrm{~nm}$ long-path filter.

2.4. Confocal Imaging of NO. To measure intracellular NO concentration, the cardiac cells were loaded with $2 \mu \mathrm{M}$ DAFFM [29]. The green fluorescence was excited at $488 \mathrm{~nm}$ and imaged through a $525 \mathrm{~nm}$ long-path filter.

2.5. Cell Viability Assay. The cell viability was assessed by propidium iodide fluorometry using a flow cytometry (FACscalibur, Becton Dickinson, NJ). Fluorescence intensity was measured at the excitation and emission wavelengths of 488 and $590 \mathrm{~nm}$, respectively. Cells were incubated in the standard Tyrode solution containing (in $\mathrm{mM}$ ) $140 \mathrm{NaCl}, 6$ $\mathrm{KCl}, 1 \mathrm{MgCl}_{2}, 1 \mathrm{CaCl}_{2}, 5$ HEPES, and 5.8 glucose (pH 7.4) for $2 \mathrm{~h}$ before the experiments. Cells were then subjected to $90 \mathrm{~min}$ of simulated ischemia followed by $30 \mathrm{~min}$ of reperfusion.

2.6. Western Blotting Analysis. Equal amount of protein lysates were loaded and electrophoresed on SDSpolyacrylamide gel and transferred to a polyvinylidene difluoride (PVDF) membrane. The membranes were probed with primary antibodies that recognize the phosphorylation of GSK-3 $\beta$, VASP, and Akt. Each primary antibody binding was detected with a secondary antibody and visualized by the enhanced chemiluminescence (ECL) method. The ECL-image was captured with Biospectrum Imaging System (UVP, Upland, USA). Equal loading of samples was confirmed by reprobing membranes with antitubulin or total protein antibodies.

2.7. Experimental Protocols. Cultured cells were washed twice with PBS and then incubated in Tyrode solution for $2 \mathrm{~h}$ prior to experiments. To examine the effect of astragaloside IV on GSK-3 $\beta$ phosphorylation at $\operatorname{Ser}^{9}$ (or Akt at $\operatorname{Ser}^{473}$ and VASP at $\operatorname{Ser}^{239}$ ), cells were exposed to a range of astragaloside IV concentrations $(20-100 \mu \mathrm{M})$ for $20 \mathrm{~min}$. The inhibitors (LY, KT, ODQ) were applied $10 \mathrm{~min}$ before exposure to astragaloside IV $(50 \mu \mathrm{M})$. In the study evaluating the effect of astragaloside IV on $\Delta \Psi_{m}$, cells were exposed to $500 \mu \mathrm{M}$ $\mathrm{H}_{2} \mathrm{O}_{2}$ for 20 min to cause mitochondrial oxidant damage. Astragaloside IV $(20-100 \mu \mathrm{M})$ was given 20 min before exposure to $\mathrm{H}_{2} \mathrm{O}_{2}$. Inhibitors (LY, KT, ODQ, L-NAME) were given $10 \mathrm{~min}$ before the exposure to astragaloside IV. In the experiments monitoring changes in intracellular NO levels, astragaloside IV was given immediately after baseline $\left(0^{\prime}\right)$ measurements, whereas the inhibitors (LY, L-NAME) were applied $10 \mathrm{~min}$ before the application of astragaloside IV. To test the effect of astragaloside IV on ischemia/reperfusion injury, cells were exposed to a simulated ischemia solution (glucose-free Tyrode solution containing $10 \mathrm{mM}$ 2-deoxy-Dglucose and $10 \mathrm{mM}$ sodium ithionite) for $90 \mathrm{~min}$ followed by $30 \mathrm{~min}$ of reperfusion with the normal Tyrode solution. Astragaloside IV was applied at the onset of reperfusion for 30 min or during ischemia (90 min) only. All chemicals were dissolved in DMSO except L-NAME (dissolved in $\mathrm{H}_{2} \mathrm{O}$ ). 

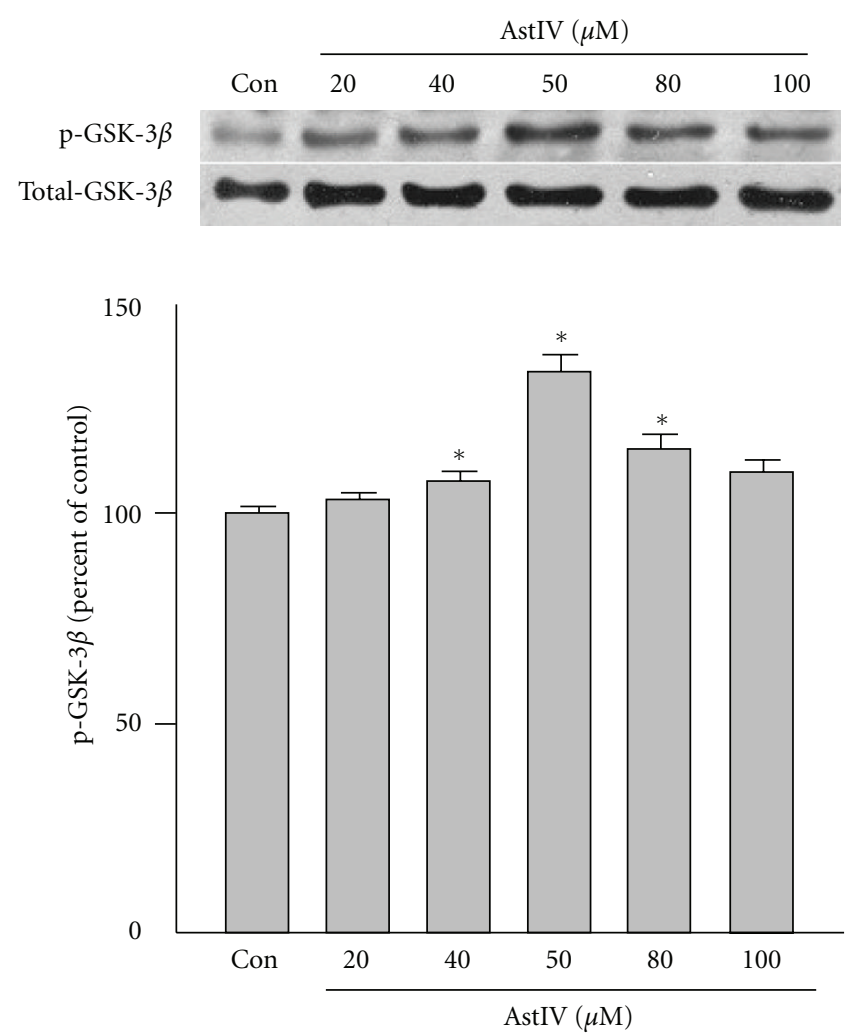

FIGURE 1: Western blotting analysis of GSK-3 $\beta$ phosphorylation at $\mathrm{Ser}^{9}$ and total GSK-3 $\beta$ protein in cardiac H9c2 cells. Astragaloside IV $(20 \sim 100 \mu \mathrm{M})$ increased GSK-3 $\beta$ phosphorylation in a dosedependent manner. Data are mean \pm SD for 6 independent experiments performed in duplicate. ${ }^{*} P<0.05$ compared to control group ( $t$-test).

2.8. Statistical Analysis. Data are expressed as mean \pm SD and obtained from at least 6 experiments. Statistical significance was determined using one-way ANOVA followed by Tukey's test. A value of $P<0.05$ was considered as statistically significant.

\section{Results}

3.1. Effects of Astragaloside IV on GSK-3 $\beta$ and Akt Phosphorylation. To determine the potential role of GSK-3 $\beta$ in the cardioprotective effect of astragaloside IV, this study first tested if astragaloside IV could enhance GSK- $3 \beta$ phosphorylation at $\mathrm{Ser}^{9}$ in $\mathrm{H} 9 \mathrm{c} 2$ cardiac cells. As shown in Figure 1, astragaloside IV significantly increased GSK-3 $\beta$ phosphorylation at Ser ${ }^{9}$ in a dose-dependent manner $(20-100 \mu \mathrm{M})$ with the peak at $50 \mu \mathrm{M}$. Thus, $50 \mu \mathrm{M}$ astragaloside IV was used in the following experiments. To define the mechanism by which astragaloside IV inactivates GSK-3 $\beta$, the experiments were carried out to test if LY294002, an inhibitor of PI3 K, can alter the action of astragaloside IV. As shown in Figure 2, the effect of astragaloside IV on GSK-3 $\beta$ phosphorylation was partially but significantly reversed by LY294002 (10 $\mu \mathrm{M})$. Moreover, astragaloside IV increased Akt phosphorylation at Ser ${ }^{473}$, an effect that was nullified by LY294002.

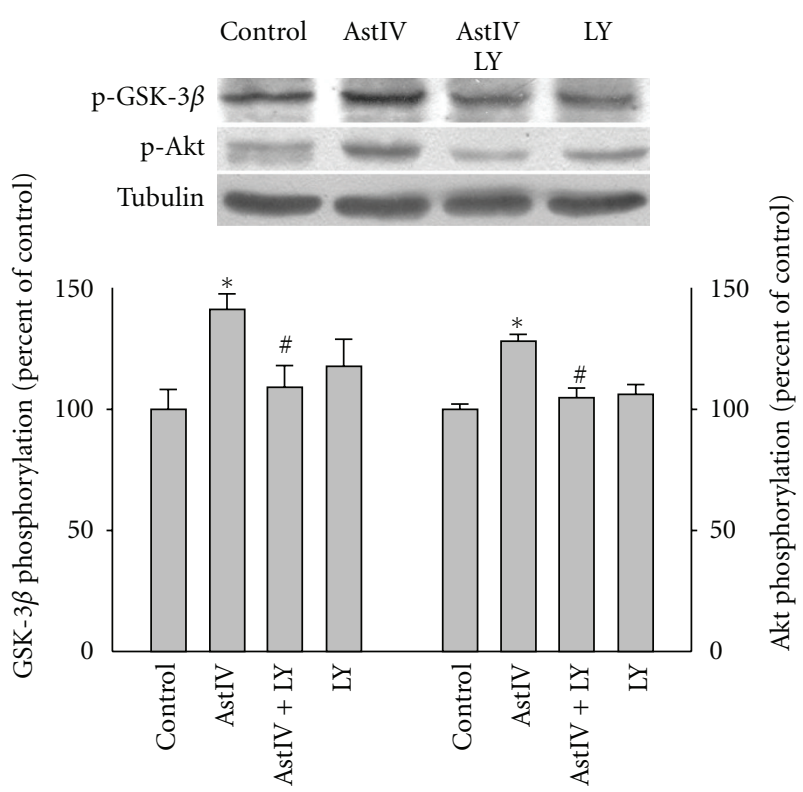

Figure 2: Western blotting analysis of GSK-3 $\beta$ at Ser $^{9}$ and Akt at $\operatorname{Ser}^{473}$ phosphorylation and Tubulin protein in cardiac H9c2 cells. Astragaloside IV $(50 \mu \mathrm{M})$ increased GSK-3 $\beta$ and Akt phosphorylation compared to control, the effect that was reversed by PI3 K inhibitor LY294002 $(10 \mu \mathrm{M})$. Data are mean \pm SD for 6 independent experiments performed in duplicate. ${ }^{*} P<0.05$ compared to control group; ${ }^{\#} P<0.05$ compared to astragaloside IV (ANOVA followed by Tukey's test).

3.2. Effect of Astragaloside IV on the mPTP Opening. To determine if astragaloside IV can prevent the MPTP opening, then experiments were conducted to determine the effect of astragaloside IV on oxidative stress-induced loss of $\Delta \Psi_{m}$ by monitoring changes in TMRE fluorescence with confocal microscopy. As shown in Figure 3, treatment of cells with $500 \mu \mathrm{M} \mathrm{H}_{2} \mathrm{O}_{2}$ induced a marked decrease in TMRE fluorescence ( $49.69 \pm 6.34 \%$ of baseline in the control group). In contrast, cells treated with 50,60 , and $80 \mu \mathrm{M}$ astragaloside IV showed much less decrease in TMRE fluorescence. To confirm that the effect of astragaloside IV on TMRE fluorescence results from the inhibition of mPTP opening but not from mitochondrial uncoupling, the mitochondrial uncoupler FCCP was applied to test its effect on TMRE fluorescence. FCCP $(0.5 \mu \mathrm{M})$ induced a marked decrease in TMRE fluorescence $(45.09 \pm 5.51 \%$ of baseline in the control group). Astragaloside IV did not change the TMRE fluorescence decrease caused by FCCP (Figure 2(S)). The GSK-3 $\beta$ inhibitor SB216763 $(3 \mu \mathrm{M})$ and the specific mPTP inhibitor cyclosporin $\mathrm{A}(0.2 \mu \mathrm{M})$ could mimic the effect of astragaloside IV to prevent the loss of TMRE fluorescence (Figure 3(S)).

3.3. The Potential Mechanisms Underlying the Inhibitory Effect of Astragaloside IV on the MPTP Opening. Figure 4 shows that astragaloside IV was not able to prevent TMRE fluorescence loss in the presence of LY294002, ODQ $(5 \mu \mathrm{M})$, a potent selective inhibitor of NO-sensitive guanylyl cyclase, 


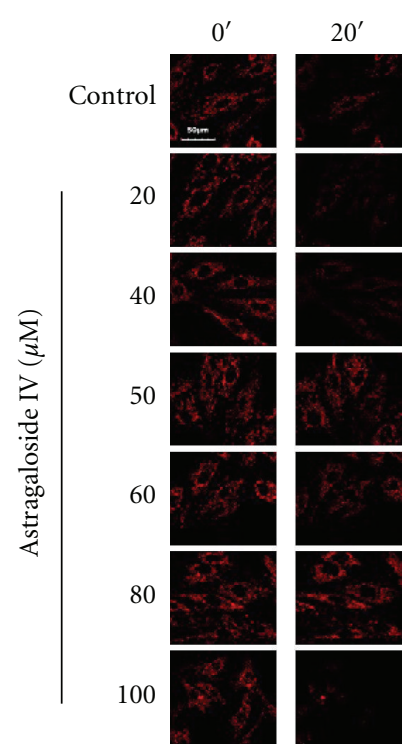

(a)

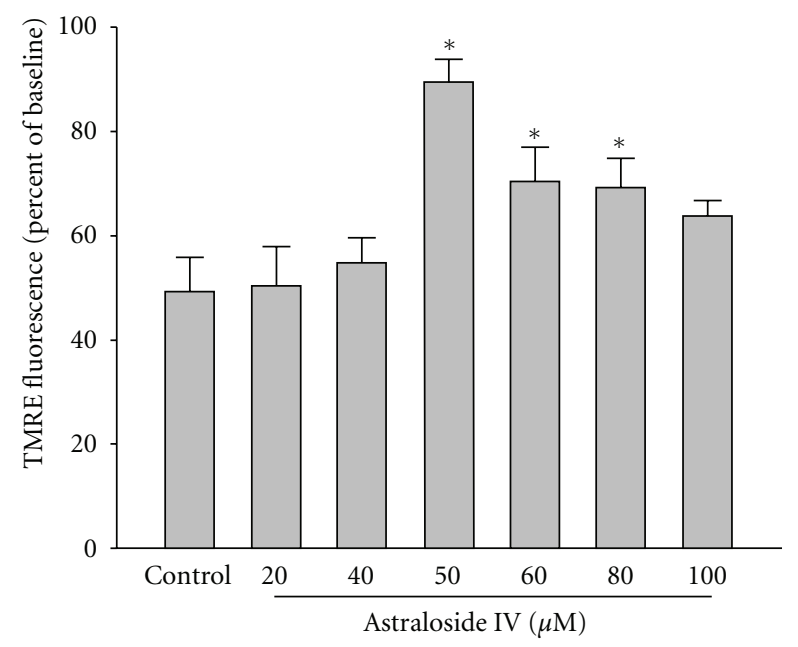

(b)

FIgURE 3: Confocal fluorescence images of TMRE at baseline and $20 \mathrm{~min}$ after exposure to $500 \mu \mathrm{M} \mathrm{H}_{2} \mathrm{O}_{2}$ in $\mathrm{H} 9 \mathrm{c} 2$ cells. (a) Astragaloside IV $(20 \sim 100 \mu \mathrm{M})$ prevented oxidant-induced TMRE fluorescence reduction in a dose-dependent manner. (b) Summarized data for TMRE fluorescence intensity measured with confocal microscopy $20 \mathrm{~min}$ after exposure to $\mathrm{H}_{2} \mathrm{O}_{2}$ expressed as a percentage of baseline. Data are mean \pm SD for 8 independent experiments performed in duplicate. ${ }^{*} P<0.05$ compared to control group ( $t$-test).

and KT5823 $(1 \mu \mathrm{M})$, a selective inhibitor of PKG. LY294002, ODQ and KT5823 alone did not change the TMRE fluorescence intensity (data not shown). In addition, the effect of astragaloside IV on GSK- $3 \beta$ phosphorylation was reversed by ODQ and KT5823. Moreover, astragaloside IV significantly increased phosphorylation of vasodilatorstimulated phosphoprotein (VASP), a substrate of PKG, and this effect was also reversed by ODQ and KT5823 (Figure 5).

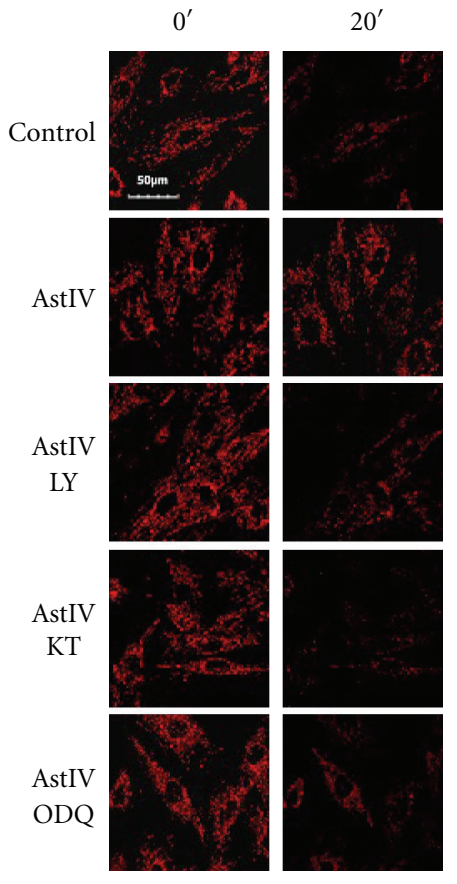

(a)

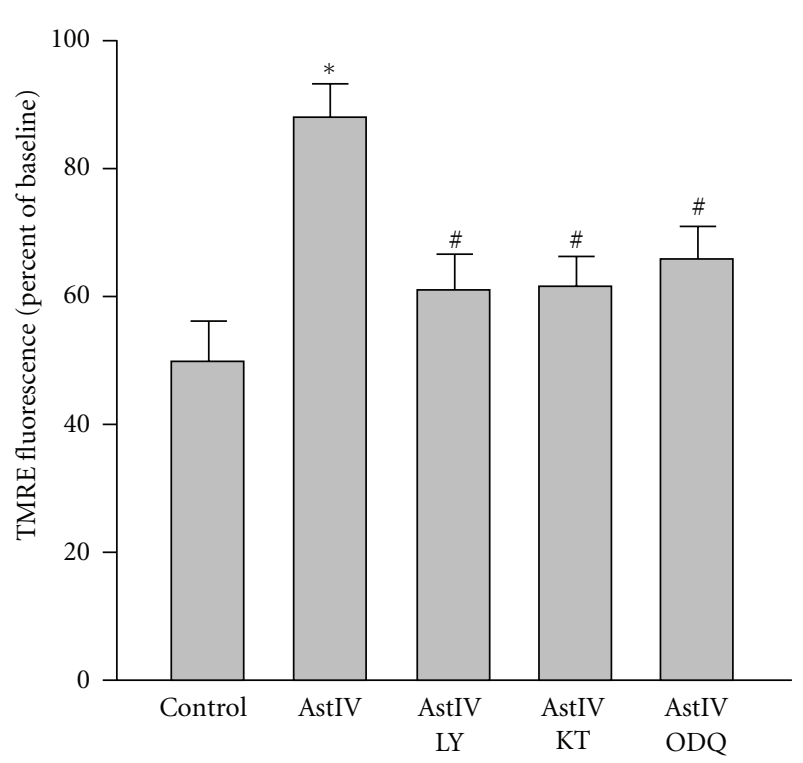

(b)

Figure 4: Confocal fluorescence images of TMRE at baseline and 20 min after exposure to $\mathrm{H}_{2} \mathrm{O}_{2}$ in cardiac $\mathrm{H} 9 \mathrm{c} 2$ cells. (a) The effect of astragaloside IV $(50 \mu \mathrm{M})$ on the oxidant-induced mPTP opening was reversed by LY294002 $(10 \mu \mathrm{M})$, the potent and selective inhibitor of NO-sensitive guanylyl cyclase ODQ, $(5 \mu \mathrm{M})$, and the specific PKG inhibitor KT5823 $(1 \mu \mathrm{M})$. (b) Summarized data for TMRE fluorescence intensity measured with confocal microscopy 20 min after exposure to $\mathrm{H}_{2} \mathrm{O}_{2}$ expressed as a percentage of baseline in cardiac H9c2 cells. Data are mean \pm SD for 6 independent experiments performed in duplicate. ${ }^{*} P<0.05$ compared to control group; ${ }^{*} P<0.05$ compared to astragaloside IV (ANOVA followed by Tukey's test). 

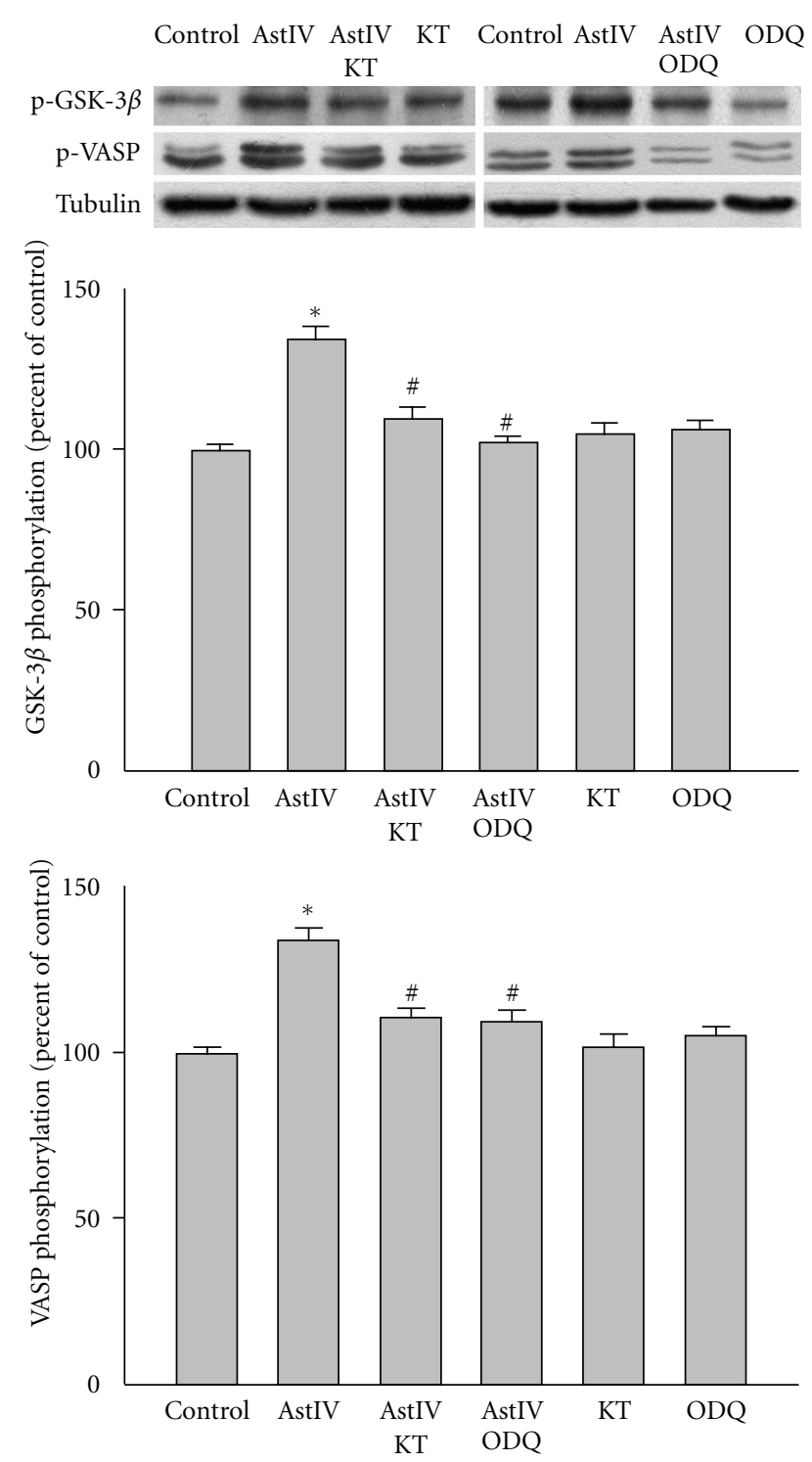

FIGURE 5: Western blotting analyses of GSK-3 $\beta$ at Ser ${ }^{9}$ and VASP at $\operatorname{Ser}^{239}$ phosphorylation in $\mathrm{H} 9 \mathrm{c} 2$ cells. Astragaloside IV $(50 \mu \mathrm{M})$ increased GSK-3 $\beta$ and VASP phosphorylation, the effect that was reversed by the potent and selective inhibitor of NOsensitive guanylyl cyclase ODQ $(5 \mu \mathrm{M})$ and the specific inhibitor of PKG KT5823 $(1 \mu \mathrm{M})$. Data are mean \pm SD for 6 independent experiments performed in duplicate. ${ }^{*} P<0.05$ compared to control group; ${ }^{\#} P<0.05$ compared to astragaloside IV (ANOVA followed by Tukey's test).

3.4. Effect of Astragaloside IV on NO Generation. To test if astragaloside IV can produce $\mathrm{NO}$ in $\mathrm{H} 9 \mathrm{c} 2$ cells, intracellular NO levels were measured by loading cells with DAFFM fluorescence. As shown in Figure 6, astragaloside IV markedly enhanced DAF-FM fluorescence intensity $20 \mathrm{~min}$ after the treatment compared to the control. The effect of astragaloside IV on NO generation was reversed by the NOS inhibitor L-NAME $(200 \mu \mathrm{M})$ and the PI3 K inhibitor LY294002. L-NAME, LY294002, and $\mathrm{H}_{2} \mathrm{O}_{2}$ alone did not change the DAF-FM fluorescence intensity.
3.5. Effect of Astragaloside IV on Cell Viability. To test the effect of astragaloside IV on ischemia/reperfusion injury, $\mathrm{H} 9 \mathrm{c} 2$ cells were subjected to $90 \mathrm{~min}$ simulated ischemia followed by $30 \mathrm{~min}$ of reperfusion. Figure 7 shows that simulated ischemia/reperfusion significantly reduced cell viability to $55.36 \pm 2.9 \%$. Astragaloside IV given during ischemia but not during reperfusion failed to improve cell viability $(55.18 \pm 3.7 \%)$. In contrast, astragaloside IV given at the onset of reperfusion for $30 \mathrm{~min}$ increased cell viability to $79.81 \pm 3.6 \%$, indicating that astragaloside IV is cardioprotective during reperfusion rather than during ischemia and thus may have a potential to save myocardium from reperfusion injury.

\section{Discussion}

This is the first study to demonstrate that astragaloside IV modulates the mPTP opening by inactivating GSK- $3 \beta$ via the PI3 K/Akt/NO/cGMP/PKG pathway. Since inhibition of the MPTP opening has been demonstrated to be a critical event in acute cardioprotection against reperfusion injury, the current finding suggests that astragaloside IV may be a promising agent to treat patients with acute myocardial infarction.

Astragaloside IV has been shown to induce cardioprotection in various experimental models $[4,5,35]$. While Ca-ATPase [8], $\mathrm{Na}(+)-\mathrm{K}(+)$-ATPase [9], ROS [10], and NO [7] have been reported to be involved in astragaloside IVinduced cardioprotection, the exact cellular and molecular events that mediate the protective effect of astragaloside IV remain to be elucidated. The $\mathrm{mPTP}$ opening has been demonstrated to be a critical determinant of myocardial ischemia/reperfusion injury [11], and the MPTP is an important target of cardioprotection [15]. The critical role of the MPTP in cardioprotection has also been demonstrated by recent reports addressing that both preconditioning and postconditioning confer cardioprotection against ischemia/reperfusion injury by inhibiting the MPTP opening $[14,16,24,36]$. In the present study, oxidative stress-induced loss of $\Delta \Psi_{m}$ was prevented by astragaloside IV, suggesting that astragaloside can modulate the MPTP opening, since the loss of $\Delta \Psi_{m}$ is caused by the mPTP opening [11]. To confirm that the effect of astragaloside IV on TMRE fluorescence results from the inhibition of mPTP opening but not from mitochondrial uncoupling, the mitochondrial uncoupler FCCP was applied to test its effect on TMRE fluorescence. FCCP induced a marked decrease in TMRE fluorescence. Astragaloside IV did not change the TMRE fluorescence decrease caused by FCCP. The observation that the selective MPTP closer cyclosporin A mimicked the effect of astragaloside IV by preventing loss of $\Delta \Psi_{m}$ further confirms the inhibitory action of astragaloside IV on the MPTP. Since a burst of reactive oxygen species upon myocardial reperfusion is associated with cardiac injury [37], our finding suggests that astragaloside IV may protect the heart from reperfusion injury by modulating the $\mathrm{mPTP}$ opening. In support, our data have shown that astragaloside IV applied at reperfusion protected cells from simulated 


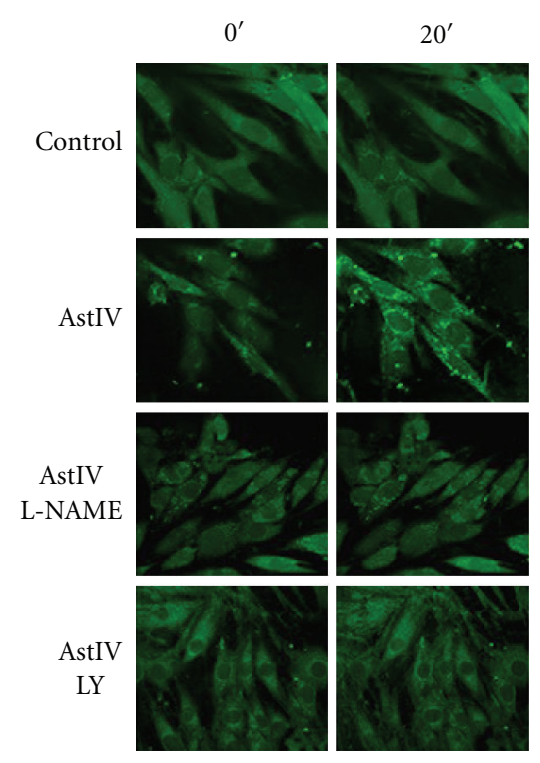

(a)

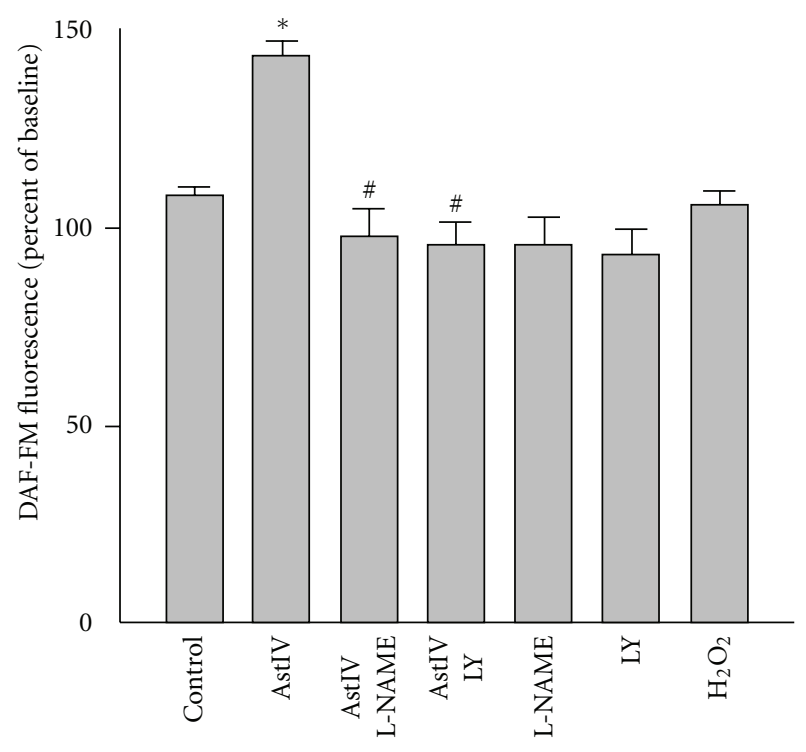

(b)

FIgURE 6: Confocal fluorescence images of DAF-FM 0 and $20 \mathrm{~min}$ after exposure to astragaloside IV in $\mathrm{H} 9 \mathrm{c} 2$ cells. (a) Astragaloside IV $(50 \mu \mathrm{M})$ significantly increased DAF fluorescence, an effect that was reversed by the NOS inhibitor L-NAME $(200 \mu \mathrm{M})$ and the PI3 K inhibitor LY294002 $(10 \mu \mathrm{M})$. (b) Summarized data for DAFFM fluorescence intensity $20 \mathrm{~min}$ after exposure to astragaloside IV expressed as a percentage of baseline. Data are mean \pm SD for 6 independent experiments performed in duplicate. ${ }^{*} P<0.05$ compared to control group; ${ }^{\#} P<0.05$ compared to astragaloside IV (ANOVA followed by Tukey's test).

ischemia/reperfusion injury, confirming the potential protective effect of astragaloside IV on reperfusion injury. Our finding also supports the prevalent notion that the MPTP is a critical and common target for various cardioprotective interventions $[38,39]$.

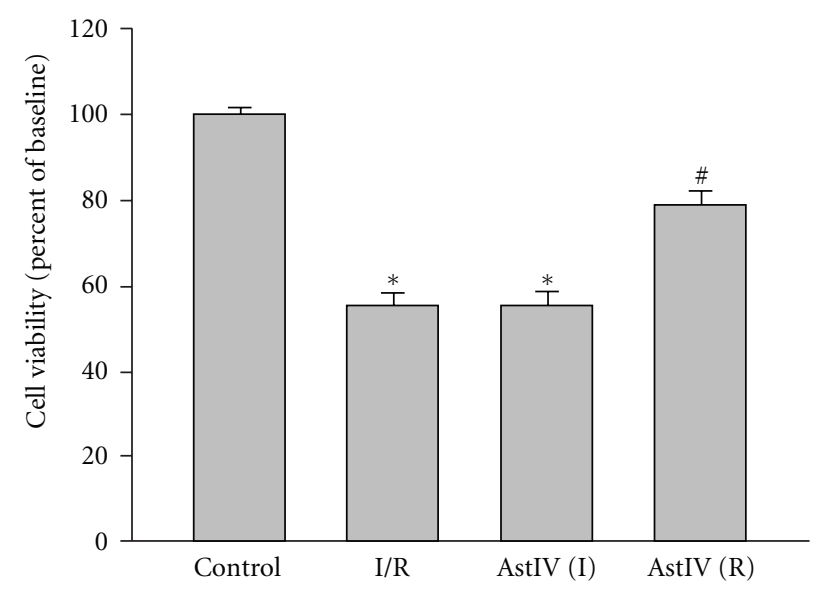

FIGURE 7: Cell viability assay in H9c2 cells subjected to $90 \mathrm{~min}$ simulated ischemia followed by $30 \mathrm{~min}$ of reperfusion. Astragaloside IV applied during ischemia only did not improve cell viability (AstIV (I)), compared with the ischemia/reperfusion control (I/R). Astragaloside IV given at reperfusion for $30 \mathrm{~min}$ significantly increased cell viability (AstIV $(\mathrm{R}))$. Data are mean \pm SD for 6 independent experiments performed in duplicate. ${ }^{*} P<0.05$ compared to the control; ${ }^{\#} P<0.05$ compared to astragaloside IV (ANOVA followed by Tukey's test).

GSK-3 $\beta$ activity is regulated by phosphorylation at its Ser $^{9}$ and $\mathrm{Tyr}^{216}$. Phosphorylation of $\mathrm{Ser}^{9}$ decreases GSK-3 $\beta$ activity, whereas phosphorylation of $\mathrm{Tyr}^{216}$ increases GSK$3 \beta$ activity [23]. GSK-3 $\beta$ is constitutively activated due to basal phosphorylation of $\mathrm{Ty}^{216}$. GSK-3 $\beta$ inactivation plays a critical role in the cardioprotective effects of ischemic preconditioning [22], morphine [23], and bradykinin [40]. GSK-3 $\beta$ was further shown to mediate the convergence of cardioprotective signaling pathways to inhibit the $\mathrm{mPTP}$ opening [24]. In support, inactivation of GSK-3 $\beta$ is crucial for prevention of the MPTP opening by preconditioning [41] and postconditioning [25]. In the present study, astragaloside IV significantly increased GSK-3 $\beta$ phosphorylation at Ser ${ }^{9}$ in a dose-dependent manner, suggesting that astragaloside IV can inactivate GSK-3 $\beta$ in cardiac cells. The selective GSK-3 $\beta$ inhibitor SB216763 could mimic the protective effect of astragaloside IV by preventing oxidative stressinduced MPTP opening. Thus, it is reasonable to propose that GSK-3 $\beta$ inactivation is critical for the preventive effect of astragaloside IV on the MPTP opening.

Activation of the cGMP/PKG signaling pathway has been proposed to lead to prevention of the MPTP opening [28, 42]. It was also reported that NO modulates the MPTP opening in mouse hearts [19]. NO has been proposed to contribute to the mechanism underlying astragaloside IVinduced cardioprotection [7]. In the present study, the action of astragaloside IV on TMRE fluorescence was reversed by a potent NO-sensitive guanylyl cyclase selective inhibitor ODQ $(5 \mu \mathrm{M})$ and a selective PKG inhibitor KT5823 $(1 \mu \mathrm{M})$, implying that the cGMP/PKG pathway may play a role in the action of astragaloside IV. In addition, the effect of astragaloside IV on GSK-3 $\beta$ phosphorylation was also 
reversed by ODQ and KT5823. Moreover, astragaloside IV significantly increased phosphorylation of vasodilatorstimulated phosphoprotein (VASP), a substrate of PKG, and this effect was again reversed by ODQ and KT5823, further confirming that the cGMP/PKG signaling pathway is required for the inhibitory action of astragaloside IV on GSK-3 $\beta$. Furthermore, astragaloside IV was also able to rapidly produce $\mathrm{NO}$ in $\mathrm{H} 9 \mathrm{c} 2$ cells. These observations strongly suggest that the NO/cGMP/PKG pathway serves as the upstream signal of GSK-3 $\beta$ inactivation in the action of astragaloside IV on the MPTP opening. In agreement with our finding, a recent study by Das et al. demonstrated that PDE5 inhibition by sildenafil induces cardioprotection by inactivating GSK-3 $\beta$ via PKG [43].

The PI3 K/Akt signaling pathway plays an important role in cardioprotection $[44,45]$ and can activate NO generation $[46,47]$. Recent studies reported that astragaloside IV promotes angiogenesis by activating the PI3 K/Akt pathway [48]. Therefore, it is possible that astragaloside IV generates NO through a pathway involving PI3 K/Akt. In the present study, astragaloside IV-induced $\mathrm{NO}$ generation was suppressed by the PI3 K inhibitor LY294002, suggesting that the PI3 K/Akt pathway may serve as the upstream signal of NO production by astragaloside IV. In addition, astragaloside IV was able to activate Akt and its inhibitory effect of GSK-3 $\beta$ was reversed by the PI3 K inhibitor LY294002. Furthermore, the effects of astragaloside IV on GSK-3 $\beta$ phosphorylation and the MPTP opening were also abrogated by LY294002. These data clearly indicate that the PI3 K/Akt signaling pathway contributes to the action of astragaloside IV by activating NO generation leading to activation of the cGMP/PKG signaling.

Since astragaloside IV protected cells from simulated ischemia/reperfusion injury when given at reperfusion, it is reasonable to propose that astragaloside IV mimicked the cardioprotective effect of postconditioning. Interestingly, the signaling elements responsible for the protective effect of astragaloside IV have also been implicated in the mechanism of preconditioning [24, 42, 49]. However, since preconditioning and postconditioning recruit similar signaling pathways at reperfusion to protect the heart from ischemia/reperfusion injury [50], it is reasonable to understand that astragaloside IV may also induce cardioprotection at reperfusion through the signaling pathways responsible for the mechanism of preconditioning.

In summary (Figure $4(\mathrm{~S})$ ), our data demonstrate that astragaloside IV prevents the MPTP opening by inactivating GSK- $3 \beta$ through the NO/cGMP/PKG signaling pathway. The PI3 K/Akt pathway activates NOS that is responsible for NO production. It should be mentioned that although the signaling pathway found here provides new insights into the mechanism by which astragaloside IV protects the heart from ischemia/reperfusion injury, some other parallel signaling pathways or elements may also be involved in the protective action of astragaloside IV. Thus, more studies are needed to fully understand the signaling mechanism underlying astragaloside IV's cardioprotection.

\section{Authors' Contribution}

Y. He and J. Xi contributed equally to this work.

\section{Acknowledgments}

This work was supported by The National Natural Science Foundation of China (NSFC 30900494), The Hebei Province Natural Science Foundation (no. H2012401019), Hebei Province Returned Overseas Scholers Science and Technology Project (no. [2011]226), and The Medical Scientific Research Project of the Health Department of Hebei Province (no. 20110168).

\section{References}

[1] Q. Yang, J. T. Lu, A. W. Zhou, B. Wang, G. W. He, and M. Z. Chen, "Antinociceptive effect of astragalosides and its mechanism of action," Acta Pharmacologica Sinica, vol. 22, no. 9, pp. 809-812, 2001.

[2] Y. Luo, Z. Qin, Z. Hong et al., "Astragaloside IV protects against ischemic brain injury in a murine model of transient focal ischemia," Neuroscience Letters, vol. 363, no. 3, pp. 218223, 2004.

[3] L. Li, H. Y. Tao, and J. B. Chen, "Anti-apoptosis effect of astragaloside on adriamycin induced rat's cardiotoxicity," Zhongguo Zhong Xi Yi Jie He Za Zhi, vol. 26, no. 11, pp. 1011$1014,2006$.

[4] Z. C. Zhang, S. J. Li, Y. Z. Yang, R. Z. Chen, J. B. Ge, and H. Z. Chen, "Effect of astragaloside on cardiomyocyte apoptosis in murine coxsackievirus B3 myocarditis," Journal of Asian Natural Products Research, vol. 9, no. 2, pp. 145-151, 2007.

[5] Y. Zhang, H. Zhu, C. Huang et al., "Astragaloside IV exerts antiviral effects against coxsackievirus B3 by upregulating interferon- $\gamma$," Journal of Cardiovascular Pharmacology, vol. 47, no. 2, pp. 190-195, 2006.

[6] H. Qi, L. Wei, Y. Han, Q. Zhang, A. S. Y. Lau, and J. Rong, "Proteomic characterization of the cellular response to chemopreventive triterpenoid astragaloside IV in human hepatocellular carcinoma cell line HepG2," International Journal of Oncology, vol. 36, no. 3, pp. 725-735, 2010.

[7] W. D. Zhang, H. Chen, C. Zhang, R. H. Liu, H. L. Li, and H. Z. Chen, "Astragaloside IV from Astragalus membranaceus shows cardioprotection during myocardial ischemia in vivo and in vitro," Planta Medica, vol. 72, no. 1, pp. 4-8, 2006.

[8] X. L. Xu, X. J. Chen, H. Ji et al., "Astragaloside IV improved intracellular calcium handling in hypoxia-reoxygenated cardiomyocytes via the sarcoplasmic reticulum Ca 2+-ATPase," Pharmacology, vol. 81, no. 4, pp. 325-332, 2008.

[9] J. Y. Zhou, Y. Fan, J. L. Kong, D. Z. Wu, and Z. B. Hu, "Effects of components isolated from Astragalus membranaceus Bunge on cardiac function injured by myocardial ischemia reperfusion in rats," Zhongguo Zhong Yao Za Zhi, vol. 25, no. 5, pp. 300-302, 2000.

[10] J. Y. Hu, J. Han, Z. G. Chu et al., "Astragaloside IV attenuates hypoxia-induced cardiomyocyte damage in rats by upregulating superoxide dismutase-1 levels," Clinical and Experimental Pharmacology and Physiology, vol. 36, no. 4, pp. 351-357, 2009.

[11] J. N. Weiss, P. Korge, H. M. Honda, and P. Ping, "Role of the mitochondrial permeability transition in myocardial disease," Circulation Research, vol. 93, no. 4, pp. 292-301, 2003. 
[12] M. S. Suleiman, A. P. Halestrap, and E. J. Griffiths, "Mitochondria: a target for myocardial protection," Pharmacology and Therapeutics, vol. 89, no. 1, pp. 29-46, 2001.

[13] L. Argaud, O. Gateau-Roesch, D. Muntean et al., "Specific inhibition of the mitochondrial permeability transition prevents lethal reperfusion injury," Journal of Molecular and Cellular Cardiology, vol. 38, no. 2, pp. 367-374, 2005.

[14] L. Argaud, O. Gateau-Roesch, O. Raisky, J. Loufouat, D. Robert, and M. Ovize, "Postconditioning inhibits mitochondrial permeability transition," Circulation, vol. 111, no. 2, pp. 194-197, 2005.

[15] A. P. Halestrap, S. J. Clarke, and S. A. Javadov, "Mitochondrial permeability transition pore opening during myocardial reperfusion-a target for cardioprotection," Cardiovascular Research, vol. 61, no. 3, pp. 372-385, 2004.

[16] D. J. Hausenloy, H. L. Maddock, G. F. Baxter, and D. M. Yellon, "Inhibiting mitochondrial permeability transition pore opening: a new paradigm for myocardial preconditioning?" Cardiovascular Research, vol. 55, no. 3, pp. 534-543, 2002.

[17] D. J. Hausenloy, M. R. Duchen, and D. M. Yellon, "Inhibiting mitochondrial permeability transition pore opening at reperfusion protects against ischaemia-reperfusion injury," Cardiovascular Research, vol. 60, no. 3, pp. 617-625, 2003.

[18] S. A. Javador, S. Clarke, M. Das, E. J. Griffiths, K. H. H. Lim, and A. P. Halestrap, "Ischaemic preconditioning inhibits opening of mitochondrial permeability transition pores in the reperfused rat heart," Journal of Physiology, vol. 549, no. 2, pp. 513-524, 2003.

[19] G. Wang, D. A. Liem, T. M. Vondriska et al., "Nitric oxide donors protect murine myocardium against infarction via modulation of mitochondrial permeability transition," American Journal of Physiology, vol. 288, no. 3, pp. H1290H1295, 2005.

[20] P. Cohen and S. Frame, "The renaissance of GSK3," Nature Reviews Molecular Cell Biology, vol. 2, no. 10, pp. 769-776, 2001.

[21] S. Frame and P. Cohen, "GSK3 takes centre stage more than 20 years after its discovery," Biochemical Journal, vol. 359, no. 1, pp. 1-16, 2001.

[22] H. Tong, K. Imahashi, C. Steenbergen, and E. Murphy, "Phosphorylation of glycogen synthase kinase- $3 \beta$ during preconditioning through a phosphatidylinositol-3-kinase-dependent pathway is cardioprotective," Circulation Research, vol. 90, no. 4, pp. 377-379, 2002.

[23] E. R. Gross, A. K. Hsu, and G. J. Gross, "Opioid-induced cardioprotection occurs via glycogen synthase kinase b inhibition during reperfusion in intact rat hearts," Circulation Research, vol. 94, no. 7, pp. 960-966, 2004.

[24] M. Juhaszova, D. B. Zorov, S. H. Kim et al., "Glycogen synthase kinase- $3 \beta$ mediates convergence of protection signalling to inhibit the mitochondrial permeability transition pore," The Journal of Clinical Investigation, vol. 113, no. 11, pp. 15351549, 2004.

[25] L. Gomez, M. Paillard, H. Thibault, G. Derumeaux, and M. Ovize, "Inhibition of GSK $3 \beta$ by postconditioning is required to prevent opening of the mitochondrial permeability transition pore during reperfusion," Circulation, vol. 117, no. 21, pp. 2761-2768, 2008.

[26] K. Zhou, L. Zhang, J. Xi, W. Tian, and Z. Xu, "Ethanol prevents oxidant-induced mitochondrial permeability transition pore opening in cardiac cells," Alcohol and Alcoholism, vol. 44, no. 1, pp. 20-24, 2009.

[27] J. Xi, H. Wang, R. A. Mueller, E. A. Norfleet, and Z. Xu, "Mechanism for resveratrol-induced cardioprotection against reperfusion injury involves glycogen synthase kinase $3 \beta$ and mitochondrial permeability transition pore," European Journal of Pharmacology, vol. 604, no. 1-3, pp. 111-116, 2009.

[28] J. Xi, W. Tian, L. Zhang, Y. Jin, and Z. Xu, "Morphine prevents the mitochondrial permeability transition pore opening through $\mathrm{NO} / \mathrm{cGMP} / \mathrm{PKG} / \mathrm{Zn}^{2+} / \mathrm{GSK}-3 \beta$ signal pathway in cardiomyocytes," American Journal of Physiology, vol. 298, no. 2, pp. H601-H607, 2010.

[29] Z. Xu, S. S. Park, R. A. Mueller, R. C. Bagnell, C. Patterson, and P. G. Boysen, "Adenosine produces nitric oxide and prevents mitochondrial oxidant damage in rat cardiomyocytes," Cardiovascular Research, vol. 65, no. 4, pp. 803-812, 2005.

[30] T. Nishikawa, D. Edelstein, X. L. Du et al., "Normalizing mitochondrial superoxide production blocks three pathways of hyperglycaemic damage," Nature, vol. 404, no. 6779, pp. 787-790, 2000.

[31] R. C. Scaduto and L. W. Grotyohann, "Measurement of mitochondrial membrane potential using fluorescent rhodamine derivatives," Biophysical Journal, vol. 76, no. 1, pp. 469-477, 1999.

[32] M. Akao, B. O’Rourke, Y. Teshima, J. Seharaseyon, and E. Marbán, "Mechanistically distinct steps in the mitochondrial death pathway triggered by oxidative stress in cardiac myocytes," Circulation Research, vol. 92, no. 2, pp. 186-194, 2003.

[33] S. P. Jones, Y. Teshima, M. Akao, and E. Marbán, "Simvastatin attenuates oxidant-induced mitochondrial dysfunction in cardiac myocytes," Circulation Research, vol. 93, no. 8, pp. 697699, 2003.

[34] K. Förster, I. Paul, N. Solenkova et al., "NECA at reperfusion limits infarction and inhibits formation of the mitochondrial permeability transition pore by activating p70S6 kinase," Basic Research in Cardiology, vol. 101, no. 4, pp. 319-326, 2006.

[35] W. Zhang, C. Zhang, R. Liu et al., "Quantitative determination of Astragaloside IV, a natural product with cardioprotective activity, in plasma, urine and other biological samples by HPLC coupled with tandem mass spectrometry," Journal of Chromatography B, vol. 822, no. 1-2, pp. 170-177, 2005.

[36] D. J. Hausenloy, D. M. Yellon, S. Mani-Babu, and M. R. Duchen, "Preconditioning protects by inhibiting the mitochondrial permeability transition," American Journal of Physiology, vol. 287, no. 2, pp. H841-H849, 2004.

[37] L. B. Becker, "New concepts in reactive oxygen species and cardiovascular reperfusion physiology," Cardiovascular Research, vol. 61, no. 3, pp. 461-470, 2004.

[38] D. J. Hausenloy, S. B. Ong, and D. M. Yellon, "The mitochondrial permeability transition pore as a target for preconditioning and postconditioning," Basic Research in Cardiology, vol. 104, no. 2, pp. 189-202, 2009.

[39] F. Di Lisa and P. Bernardi, "Mitochondria and ischemiareperfusion injury of the heart: fixing a hole," Cardiovascular Research, vol. 70, no. 2, pp. 191-199, 2006.

[40] S. S. Park, H. Zhao, R. A. Mueller, and Z. Xu, "Bradykinin prevents reperfusion injury by targeting mitochondrial permeability transition pore through glycogen synthase kinase $3 \beta$," Journal of Molecular and Cellular Cardiology, vol. 40, no. 5, pp. 708-716, 2006.

[41] M. Nishihara, T. Miura, T. Miki et al., "Modulation of the mitochondrial permeability transition pore complex in GSK$3 \beta$-mediated myocardial protection," Journal of Molecular and Cellular Cardiology, vol. 43, no. 5, pp. 564-570, 2007.

[42] A. D. T. Costa, S. V. Pierre, M. V. Cohen, J. M. Downey, and K. D. Garlid, "cGMP signalling in pre- and post-conditioning: 
the role of mitochondria," Cardiovascular Research, vol. 77, no. 2, pp. 344-352, 2008.

[43] A. Das, L. Xi, and R. C. Kukreja, "Protein kinase Gdependent cardioprotective mechanism of phosphodiesterase5 inhibition involves phosphorylation of ERK and GSK $3 \beta$," The Journal of Biological Chemistry, vol. 283, no. 43, pp. 29572-29585, 2008.

[44] S. M. Davidson, D. Hausenloy, M. R. Duchen, and D. M. Yellon, "Signalling via the reperfusion injury signalling kinase (RISK) pathway links closure of the mitochondrial permeability transition pore to cardioprotection," International Journal of Biochemistry and Cell Biology, vol. 38, no. 3, pp. 414-419, 2006.

[45] D. J. Hausenloy and D. M. Yellon, "New directions for protecting the heart against ischaemia-reperfusion injury: targeting the Reperfusion Injury Salvage Kinase (RISK)pathway," Cardiovascular Research, vol. 61, no. 3, pp. 448-460, 2004.

[46] D. Fulton, J. P. Gratton, T. J. McCabe et al., "Regulation of endothelium-derived nitric oxide production by the protein kinase Akt," Nature, vol. 399, no. 6736, pp. 597-601, 1999.

[47] S. Dimmeler, I. Fleming, B. Fisslthaler, C. Hermann, R. Busse, and A. M. Zeiher, "Activation of nitric oxide synthase in endothelial cells by Akt- dependent phosphorylation," Nature, vol. 399, no. 6736, pp. 601-605, 1999.

[48] L. Zhang, Q. Liu, L. Lu, X. Zhao, X. Gao, and Y. Wang, "Astragaloside IV stimulates angiogenesis and increases hypoxiainducible factor- $1 \alpha$ accumulation via phosphatidylinositol 3kinase/akt pathway," Journal of Pharmacology and Experimental Therapeutics, vol. 338, no. 2, pp. 485-491, 2011.

[49] D. J. Hausenloy, A. Tsang, M. M. Mocanu, and D. M. Yellon, "Ischemic preconditioning protects by activating prosurvival kinases at reperfusion," American Journal of Physiology, vol. 288, no. 2, pp. H971-H976, 2005.

[50] D. J. Hausenloy and D. M. Yellon, "Preconditioning and postconditioning: united at reperfusion," Pharmacology and Therapeutics, vol. 116, no. 2, pp. 173-191, 2007. 


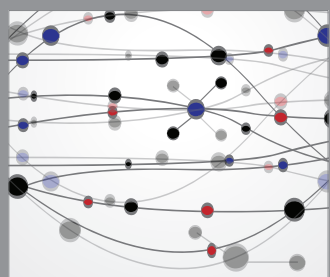

The Scientific World Journal
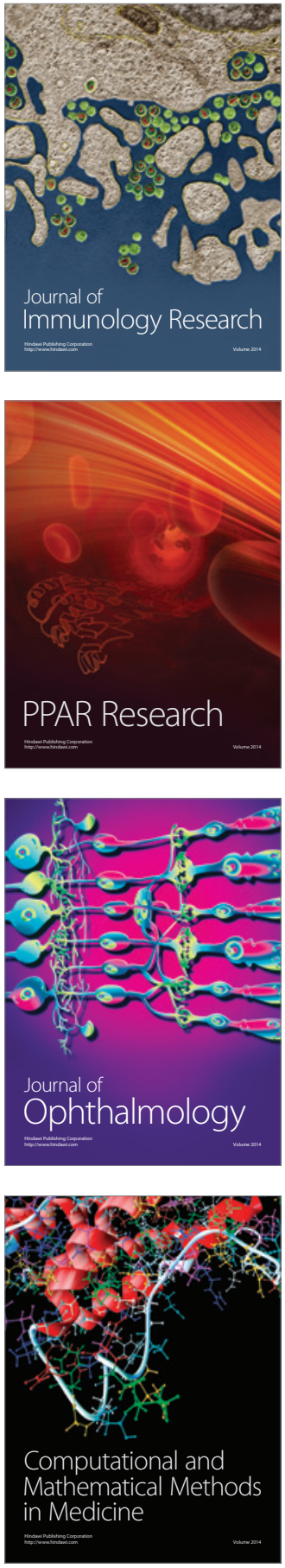

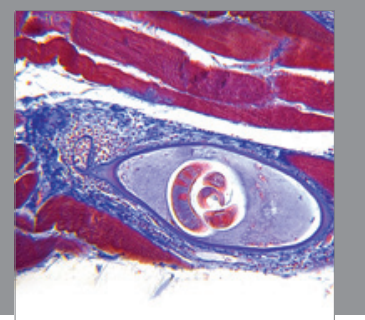

Gastroenterology

Research and Practice
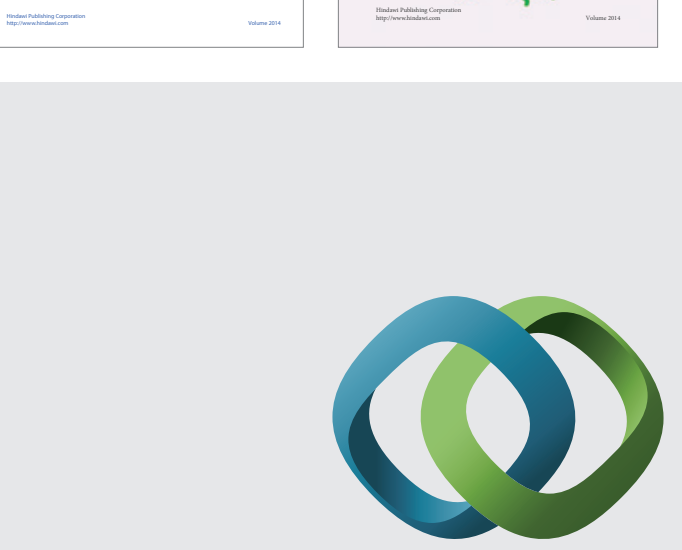

\section{Hindawi}

Submit your manuscripts at

http://www.hindawi.com
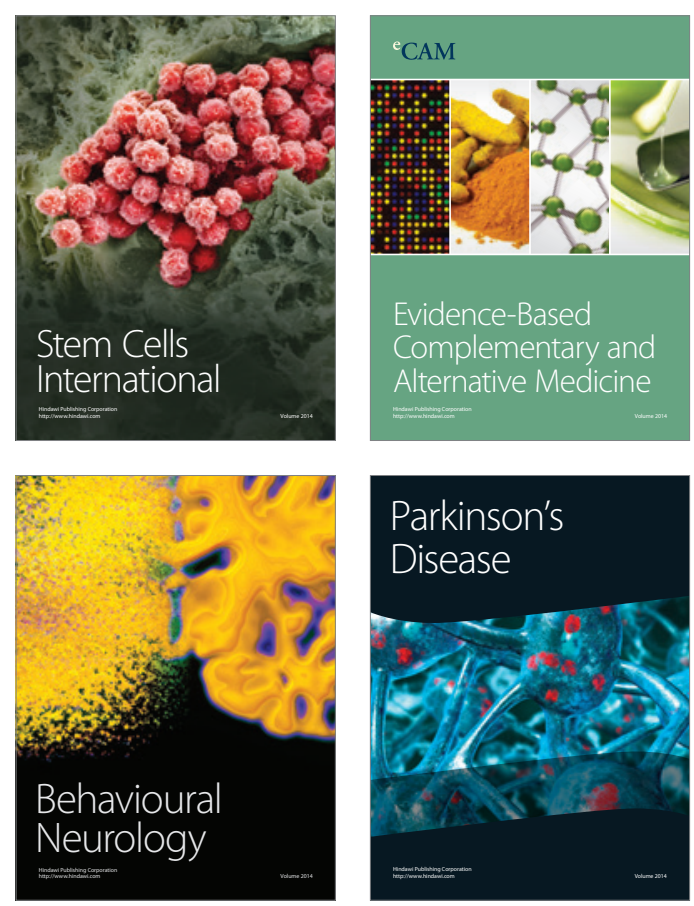

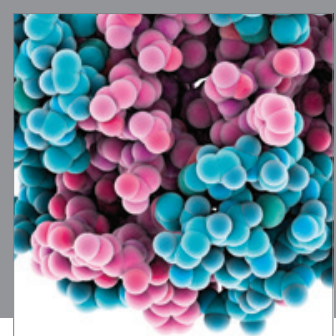

Journal of
Diabetes Research

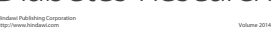

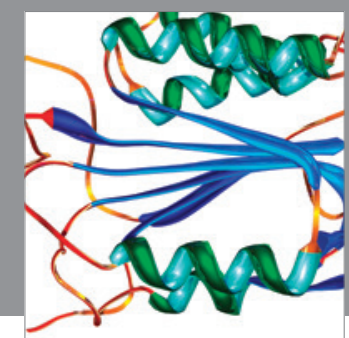

Disease Markers
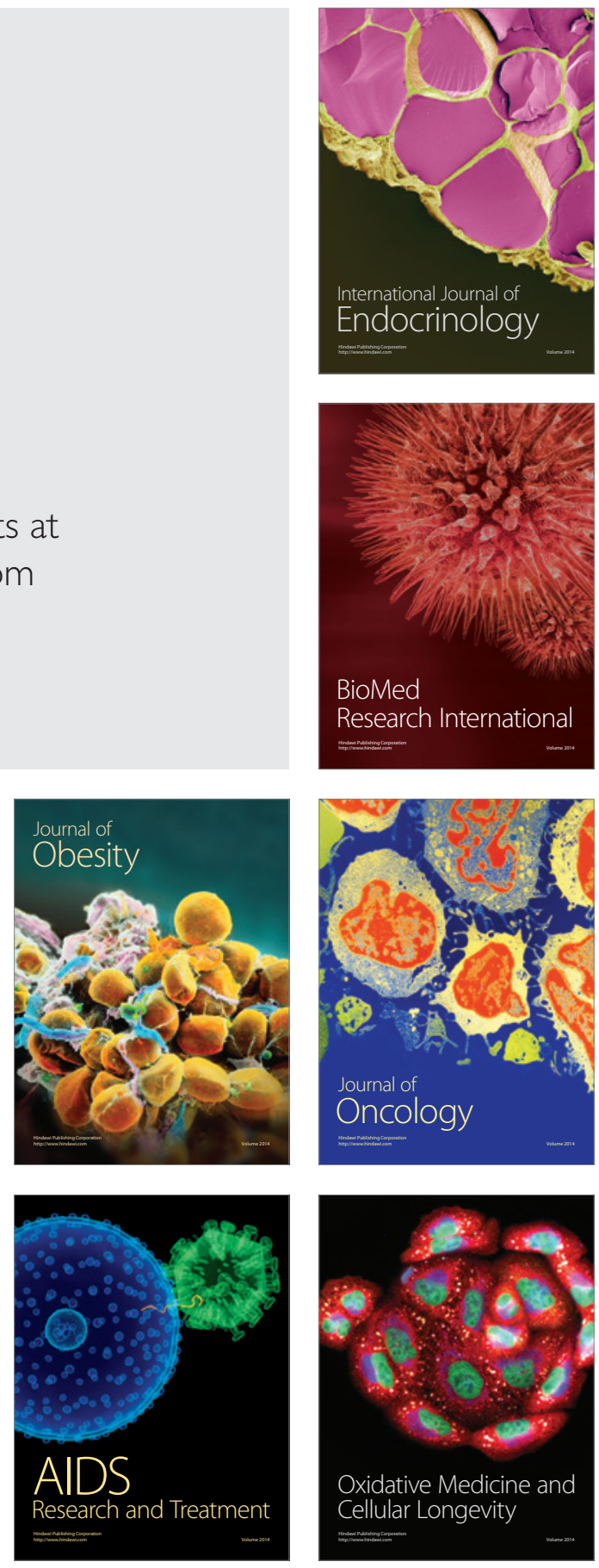